\title{
New generic records of grasses from Tripura, India
}

\author{
Sampa Ghosh \& Debjyoti Bhattacharyya* \\ Plant Taxonomy and Biosystematics Laboratory, Department of Life Science and Bioinformatics, Assam University, Silchar 788 011, India \\ *Email: dbhattacharyya_aus@yahoo.in
}

\section{ARTICLE HISTORY}

Received: 03 March 2020

Accepted: 05 May 2020

Published: 01 July 2020

\section{KEYWORDS}

Eriochloa; Gramineae; Heteropogon;

monocotyledons; northeastern

India; Perotis; Phalaris; Poaceae;

Pseudoraphis

\section{ABSTRACT}

Five species of grasses (Gramineae, nom. alt. Poaceae) namely Eriochloa procera (Retz.) C. E. Hubb., Heteropogon contortus (L.) P. Beauv. ex Roem. \& Schult., Perotis indica (L.) Kuntze, Phalaris minor Retz. and Pseudoraphis brunoniana (Griff.) Pilg. are reported here for the first time from Tripura, India. Literature review revealed that all are the first representative species under respective genus from the state which eventually proclaim five new generic records of grasses from the state of Tripura. Brief description with illustration, habitat, phenology of all the species are presented. Field photographs are also given for facilitating easy identification.

\section{Introduction}

Poaceae (nom. alt. Gramineae) are the fifth largest family among the angiosperms in the world (1) and are represented by more than 10000 species under 715 genera (2). In India, the family is the largest with about 1225 species under 268 genera (3). According to a comparatively recent report (4), there are 1291 species under 263 genera in the country. Northeastern part of India is represented with 146 genera and 475 species (5). The state Tripura harbours 118 species of grasses under 60 genera (5-9).

During field visits in the state of Tripura since 2014, the first author (SG) had collected some noteworthy grass specimens from different ecoclimatic regions of the state. After critical morphological analysis and scrutiny of relevant literature $(5,10-13)$, the species were identified as Eriochloa procera (Retz.) C. E. Hubb., Heteropogon contortus (L.) P. Beauv. ex Roem. \& Schult., Perotis indica (L.) Kuntze, Phalaris minor Retz. and Pseudoraphis brunoniana (Griff.) Pilg. The representative herbarium specimens of these species housed in ASSAM and CAL were also examined for preliminary identification of specimens. The digital images of Type specimens available at Kew Herbarium Catalogue were also perused for confirmation of the identity of the specimens. The review of pertinent literature $(3,6,7,10,11,13)$ revealed that all these five species have not been hitherto reported from the state of Tripura, hence, this communication reports the occurrence of these five species of grasses as new records from the state for the first time. Table 1 shows the distribution of all these species in different states of north-eastern India.

\section{Materials and Methods}

Following conventional morpho-taxonomical method of research, the present study includes extensive and intensive field surveys in the study area, collection of fresh plant specimens, preservation of specimens and critical morpho-taxonomic investigation. After collection, the specimens were dissected and studied under stereo zoom dissecting microscope as well as compound microscope. Identification was done following keys available in different authentic literature $(5,10-13)$. Matching of materials with the authentic specimens housed in ASSAM and CAL was carried out. Scrutiny of digital images of Type specimens available at Kew Herbarium Catalogue was also made. International Plant Names Index (IPNI) (http://www.ipni.org) (14) was cited for correct nomenclature. Voucher specimens have been deposited in the departmental herbarium of Assam University, Silchar.

(c) Ghosh \& Bhattacharyya (2020). This is an open-access article distributed under the terms of the Creative Commons Attribution License, which permits unrestricted use, distribution, and reproduction in any medium, provided the original author and source are credited (https://creativecommons.org/licenses/by/4.0/).

To cite this article: Ghosh S, Bhattacharyya D. New generic records of grasses from Tripura, India. Plant Science Today. 2020; 7(3): 319-332. https://doi.org/10.14719/pst.2020.7.3.763 
Table 1. Distribution of species of the five genera in north-eastern states of India

\begin{tabular}{|c|c|c|c|c|c|c|c|c|}
\hline Genera & $\begin{array}{l}\text { Arunachal } \\
\text { Pradesh }\end{array}$ & Assam & Manipur & Meghalaya & Mizoram & Nagaland & Sikkim & \begin{tabular}{|l|} 
Tripura \\
(Present \\
Report) \\
\end{tabular} \\
\hline $\begin{array}{l}\text { Eriochloa } \\
\text { Kunth }\end{array}$ & - & E. procera & $\begin{array}{l}01 \\
\text { E. procera }\end{array}$ & - & $\begin{array}{l}01 \\
\text { E. procera }\end{array}$ & $\begin{array}{l}01 \\
\text { E. procera }\end{array}$ & $\begin{array}{l}01 \\
\text { E. procera }\end{array}$ & $\begin{array}{l}01 \\
\text { E. procera }\end{array}$ \\
\hline $\begin{array}{l}\text { Heteropogon } \\
\text { Pers. }\end{array}$ & $\begin{array}{l}01 \\
\text { H. contortus }\end{array}$ & $\begin{array}{l}01 \\
\text { H. contortus }\end{array}$ & $\begin{array}{l}01 \\
\text { H. contortus }\end{array}$ & $\begin{array}{l}01 \\
\text { H. contortus }\end{array}$ & $\begin{array}{l}01 \\
\text { H. contortus }\end{array}$ & $\begin{array}{l}01 \\
\text { H. contortus }\end{array}$ & $\begin{array}{l}01 \\
\text { H. contortus }\end{array}$ & $\begin{array}{l}01 \\
\text { H. contortus }\end{array}$ \\
\hline Perotis Aiton & - & $\begin{array}{l}01 \\
P . \text { indica } \\
\end{array}$ & - & - & - & - & - & $\begin{array}{l}01 \\
P . \text { indica }\end{array}$ \\
\hline Phalaris L. & $\begin{array}{l}01 \\
P . \text { arundinacea }\end{array}$ & $\begin{array}{l}03 \\
P . \text { aquatica, } \\
\text { P. arundinacea } \\
\text { P. minor }\end{array}$ & - & $\begin{array}{l}02 \\
\text { P. } \text { arundinacea } \\
\text { var. } \text { arundinacea } \\
\text { var. picta } \\
\text { P. minor } \\
\end{array}$ & 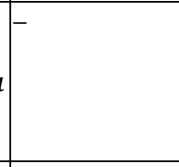 & - & $\begin{array}{l}01 \\
P . \text { arundinacea }\end{array}$ & $\begin{array}{l}01 \\
\text { P. minor }\end{array}$ \\
\hline $\begin{array}{l}\text { Pseudoraphis } \\
\text { Griff. }\end{array}$ & - & $\begin{array}{l}02 \\
P . \text { minuta, } \\
P . \text { spinescens }\end{array}$ & $\begin{array}{l}01 \\
\text { P. brunoniana }\end{array}$ & - & - & \begin{tabular}{|l|}
01 \\
$P$. spinescens
\end{tabular} & - & $\begin{array}{l}01 \\
\text { P. brunoniana }\end{array}$ \\
\hline
\end{tabular}

\section{Results and Discussion}

\section{Key to the species}

1a. Spikelets awned. . .2

1b. Spikelets un-awned. .3

2a. Awns 2-4 cm long, 1 per spikelet; racemes heterogeneous with lower homogamous and upper heterogamous spikelets....2. Heteropogon contortus

2b. Awns 6-10 mm long, 2 per spikelet; racemes homogamous only 3. Perotis indica

3a. Plants aquatic to semi-aquatic; spikelets subtended by a bristle, formed by prolongation of the branches. 5. Pseudoraphis brunoniana

3b. Plants terrestrial to partly marshy; spikelets not subtended by bristles

4a. Lower glume absent; base of the spikelet represented by a swelled callus like structure 1. Eriochloa procera

4b. Lower glume well-developed; callus like structure at the base of the spikelet absent....4. Phalaris minor

1. Eriochloa procera (Retz.) C.E. Hubb. in Bull. Misc. Inform. Kew 1930: 256. 1930; Bor, Fl. Assam 5: 268. 1940 \& Grass. Burma, Ceylon, India, Pakistan: 312. 1960; Karthik. et al., Fl. Ind. Enumerat. Monocot.: 219. 1989; Sreek. \& V. J. Nair, Fl. Kerala Grass.: 250. 1991; Shukla, Grass. N.E. India: 326. 1996; S. Moulik, Grass. Bamb. India: 102. 1997. Agrostis procera Retz., Observ. Bot. 4: 19. 1786. Fig. 1, Image 1.

Description: Annual or perennial, tufted, terrestrial to partly marshy. Culms geniculate, $80-100 \mathrm{~cm}$ high, glabrous; nodes minutely hairy. Leaf blade linearlanceolate, 9-20 × ca. $0.5 \mathrm{~cm}$, glabrous, midrib prominent; ligule 1-2 $\mathrm{mm}$, membranous with fringe of whitish hairs; leaf sheaths keeled, ciliate at one margin. Inflorescence a lax panicle, 6-20 cm long; racemes 7-11, $5-10 \mathrm{~cm}$ long; rachis triquetrous. Spikelets usually paired except raceme apex, ellipticlanceolate to lanceolate, $2.7-3 \mathrm{~mm}$ long, acute at apex, covered with soft silky hairs, greenish with tinge of purple; pedicels triquetrous, hispid; ring or cup-like disk at the base. Lower glume absent, represented by a swelling rim or collar-like callus at the base of the spikelet. Upper glume ovatelanceolate, 2.7-3 × ca. $1 \mathrm{~mm}$, chartaceous, 5-nerved, covered by soft whitish hairs, apex finely serrulate. Florets 2; lower barren, epaleate, upper bisexual. Lower lemma ovate-lanceolate, $2.5-2.7 \times 0.7-1 \mathrm{~mm}, 5$ nerved, ciliate from middle to apex, margin slightly serrulate towards apex. Lower palea absent. Upper lemma $2.3-2.5 \times 0.5-0.7 \mathrm{~mm}$, subcoriaceous, faintly 1-3-nerved, with $0.2-0.3 \mathrm{~mm}$ long arista, barbellate at apex, margin thick, inrolled, smooth. Upper palea oblong-elliptic or boat-shaped, 1.5-1.7 × ca. $0.5 \mathrm{~mm}$, subcoriaceous, faintly 2-nerved, 2-keeled, shiny, margin inrolled. Lodicules 2, ca. $0.2 \mathrm{~mm}$ long, membranous. Stamens 3, ca. $1.5 \mathrm{~mm}$ long, greenish yellow. Pistil ca. $1.5 \mathrm{~mm}$ long; style 2, filiform; stigma bifid, plumose.

Habitat: Along marshy or semi-aquatic fields.

Flowering \& Fruiting: July-October.

Specimen examined: India, Tripura, West district, West Pratapgarh, $23^{\circ} 825343 \mathrm{~N}, \quad 91^{0} 269269$ E, 14.10.2016, S. Ghosh 12766, fl.

Distribution: India [Andaman and Nicobar Islands, Andhra Pradesh, Assam, Bihar, Gujarat, Karnataka, Kerala, Maharashtra, Manipur, Mizoram, Nagaland, Odisha, Punjab, Sikkim, Tamil Nadu, Tripura (Present report), Uttar Pradesh, West Bengal]; Myanmar, Nepal, Sri Lanka, Australia, Tropical Africa $(5,13)$.

IUCN Status: Least Concern (15).

Note: This species can easily be identified by its reddish-pink coloured collar-like callus at the base of the spikelets.

2. Heteropogon contortus (L.) P. Beauv. ex Roem. \& Schult., Syst. Veg. ed. 2: 836. 1817; Bor, Fl. Assam 5: 402. 1940 \& Grass. Burma, Ceylon, India, Pakistan: 163. 1960; Patunkar, Grass. Marathwada: 66, f. 20. 1980; Karthik. et al., Fl. Ind. Enumerat. Monocot.: 226. 1989; Sreek. \& V. J. Nair, Fl. Kerala Grass.: 112. 1991; Shukla, Grass. N. E. India: 99. 1996; S. Moulik, Grass. Bamboos India: 266. 1997; Kabeer \& V. J. Nair, Fl. Tamil Nadu Grass.: 427. 2009. Andropogon contortus L., Sp. Pl.: 1045. 1753. Fig. 2, Image 2.

Description: Perennials, tufted. Culms erectgeniculate, 25-100 cm high, glabrous; nodes glaucous, 


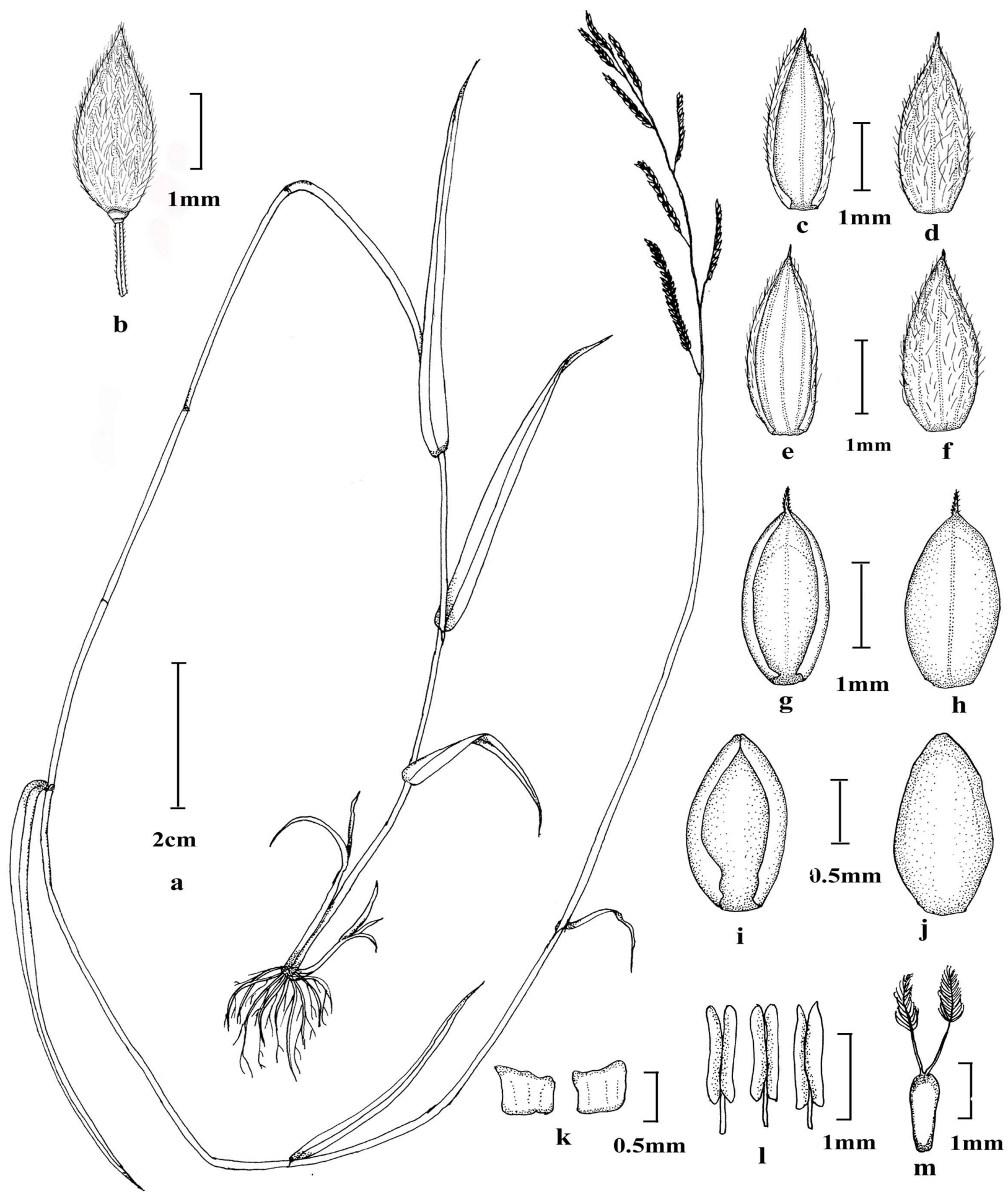

Fig. 1. Eriochloa procera (Retz.) C. E. Hubb.

a. Habit; b. Single spikelet; c. Upper glume (adaxial view); d. Upper glume (abaxial view); e. Lower lemma (adaxial view); f. Lower lemma (abaxial view); g. Upper lemma (adaxial view); h. Upper lemma (abaxial view); i. Upper palea (adaxial view); j. Upper palea (abaxial view); k. Lodicules; l. Stamens; m. Pistil.

glabrous, lower ones rooting. Leaf blades linear to narrowly lanceolate, $3-11 \times 0.2-0.5 \mathrm{~cm}$, rounded at base, serrulate at margins, acute at apex, midrib prominent, sparsely tuberculate- hairy on adaxial surface; ligule membranous, fimbriate at apex; leaf sheaths keeled on back, margin hairy. Raceme solitary, 3-7 cm long (excluding awns), decumbent, with lower homogamous spikelet pairs and upper heterogamous awned spikelet pairs, sparsely hairy, purplish green. Homogamous spikelet paired, unawned; one sessile and other pedicelled. Lower homogamous sessile spikelets linear-lanceolate, 5-6.5 $\times$ ca. $1 \mathrm{~mm}$, sparsely hairy. Lower glume linearlanceolate, 5.5-6.5 × са. $0.8 \mathrm{~mm}$, 7-9-nerved, 


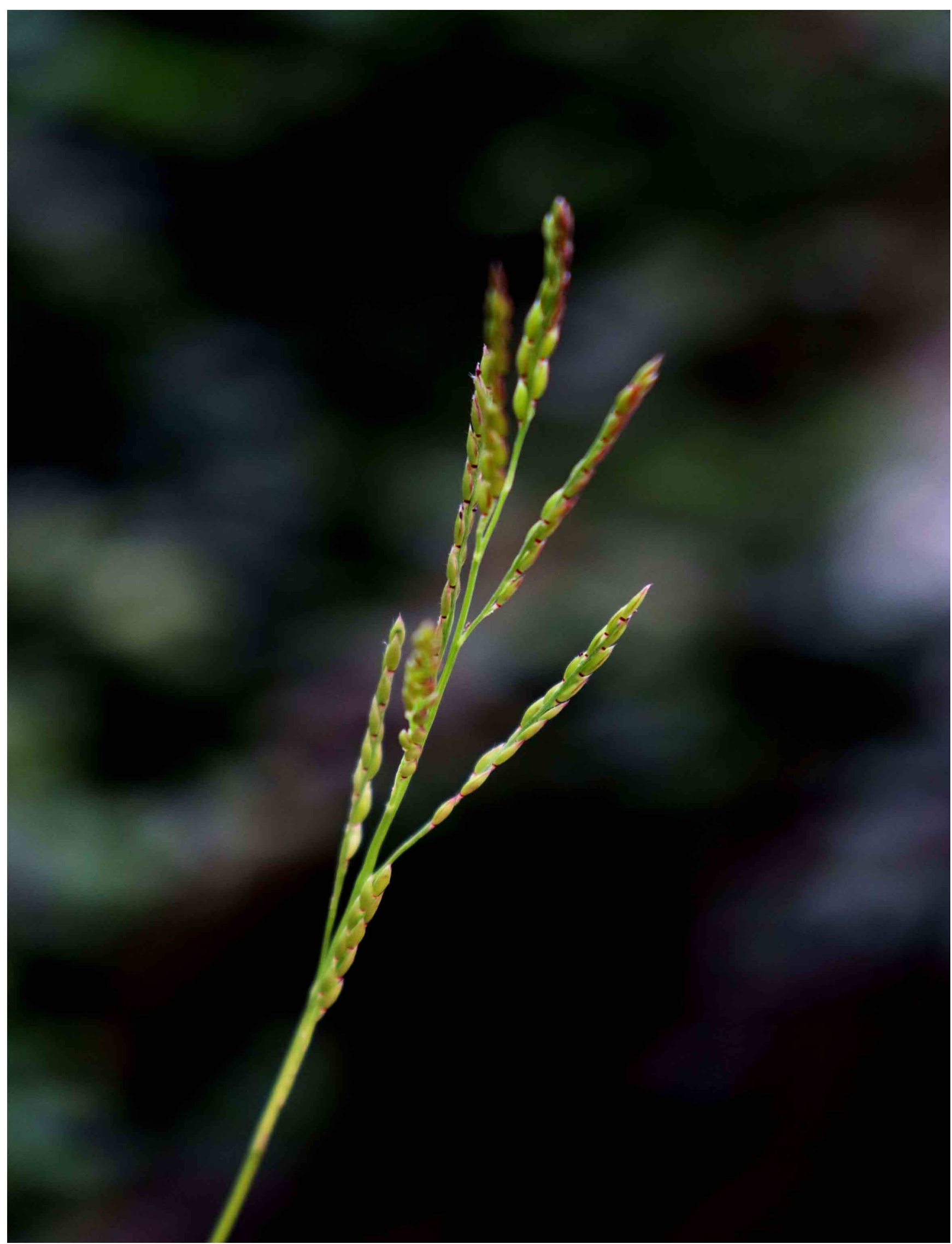

Image 1. Eriochloa procera (Retz.) C. E. Hubb.

chartaceous, incurved, tuberculate hairy on abaxial surface; 2-keeled at tip portion. Upper glume oblonglanceolate, 5-6 × ca. $0.8 \mathrm{~mm}, 1-3-$ nerved, midrib prominent, ciliate; chartaceous, inturned, ciliate hairy at margin and apex. Florets 2, both epaleate. Lower lemma oblong-lanceolate, 5-5.5 × ca. $0.8 \mathrm{~mm}$, 1-nerved, sparsely ciliate at margin and apex. Upper lemma linear-lanceolate, ca. $5 \times 0.8 \mathrm{~mm}$, slightly 
ciliate at margin and apex. Lodicules 2, 0.5-0.7 mm, 4-5.5 × ca. $1 \mathrm{~mm}$, sparsely hairy. Lower glume hyaline. Stamens 3; anthers $2-2.5 \mathrm{~mm}$ long. lanceolate, ca. $5 \times 1 \mathrm{~mm}$, faintly 7-nerved; inturned, Homogamous pedicelled spikelets linear-lanceolate, winged at apex; tuberculate hairy on adaxial surface.

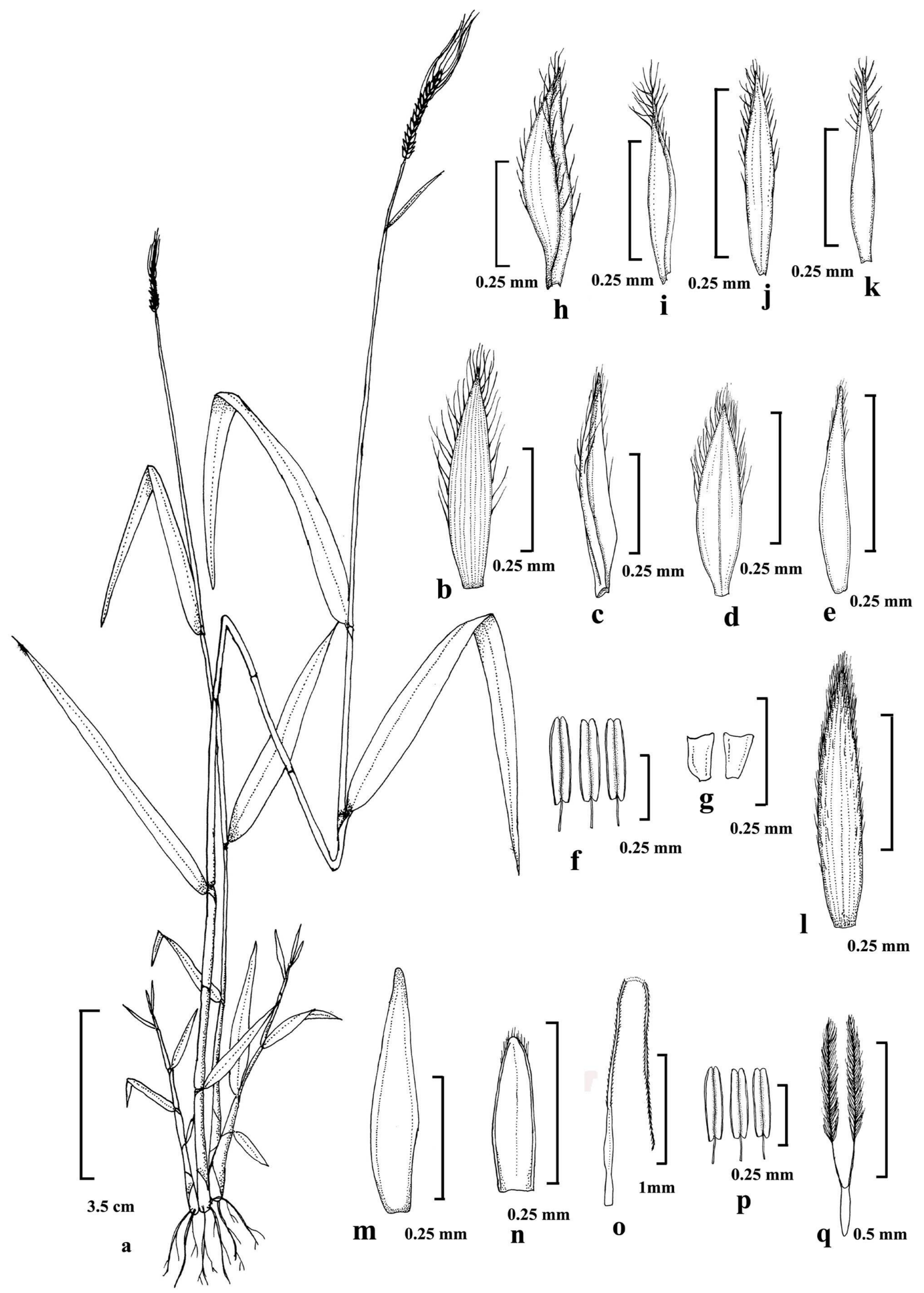

Fig. 2. Heteropogon contortus (L.) P. Beauv. ex Roem. \& Schult.

a. Habit; b-g. Lower homogamous sessile spikelet; b. Lower glume (abaxial view); c. Upper glume (side view); d. Lower lemma (abaxial view); e. Upper lemma (abaxial view); f. Stamens; g. Lodicules; h-k. Lower homogamous pedicellate spikelet; h. Lower glume (side view); i. Upper glume (side view); j. Lower lemma (abaxial view); k. Upper lemma (adaxial view); l-q. Upper heterogamous sessile spikelet; l. Lower glume (abaxial view); m. Upper glume (abaxial view); n. Lower lemma (abaxial view); o. Reduced upper lemma with awn; p. Stamens; q. Pistil. 
324 䄕 GHOSH \& BHATTACHARYYA

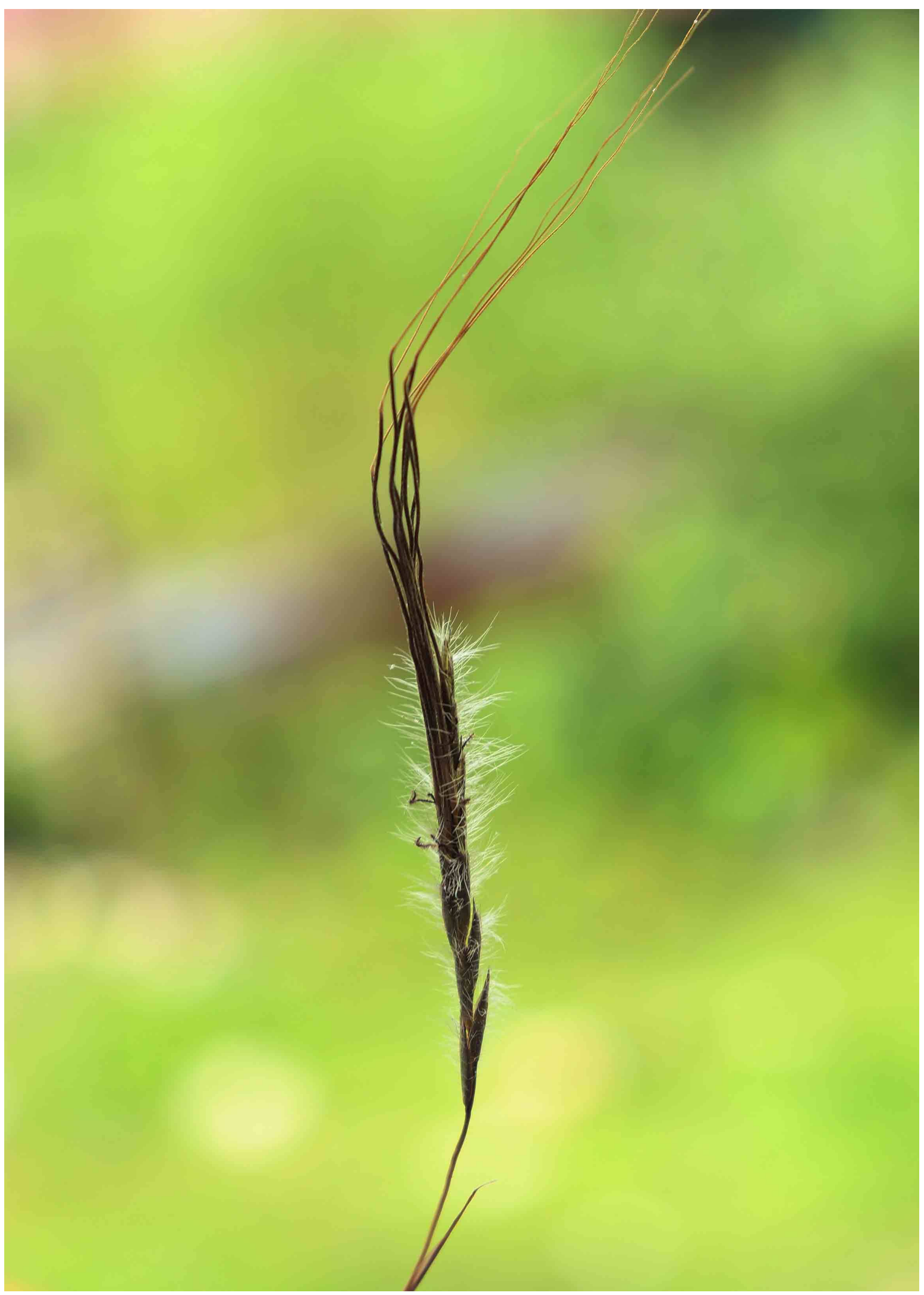

Image 2. Heteropogon contortus (L.) P. Beauv. ex Roem. \& Schult. 
Upper glume lanceolate, ca. $4.5 \times 1 \mathrm{~mm}$, ciliate at margin and apex. Florets almost similar to homogamous sessile spikelets. Stamens 3. Heterogamous sessile spikelets paired, one sessile and other pedicelled; awned. Heterogamous sessile spikelets linear-lanceolate, $4.5-6 \times$ ca. $1 \mathrm{~mm}$, awned. Lower glume oblong-lanceolate, 4.5-5.5 × ca. $1 \mathrm{~mm}$, coriaceous, 7-9 nerved, rounded at apex, pubescent on adaxial surface, greenish-brown. Upper glume linear-lanceolate, 4.5-5 × ca. $1 \mathrm{~mm}$, faintly 3-nerved; blunt at apex, sparsely pubescent on adaxial surface. Florets 2; both epaleate; lower floret empty, upper female or bisexual. Lower lemma oblong, 2-3.5 × ca. $1.3 \mathrm{~mm}$, membranous, hyaline, ciliate at apex. Upper lemma ca. $2.5 \mathrm{~mm}$ long, reduced to a hyaline awn; awn 2-4 cm long, hairy, dark brown. Pistil 2.5-3.5 $\mathrm{mm}$; style 2, filiform; stigma plumose, brown. Heterogamous pedicelled spikelets lanceolate, 4-5 × ca. $1 \mathrm{~mm}$, hairy, almost similar to homogamous pedicelled spikelets.

Habitat: On hilly slopes.

Flowering \& Fruiting: October-February.

Specimen examined: India, Tripura, West district, Dukli, Ichabazar, $23^{0} 793803 \mathrm{~N}, 91^{0} 279797 \mathrm{E}$, 04.03.2018, S. Ghosh 12930, fl.

Distribution: India (Andhra Pradesh, Arunachal Pradesh, Assam, Bihar, Gujarat, Himachal Pradesh, Karnataka, Kerala, Maharashtra, Manipur, Meghalaya, Mizoram, Nagaland, Odisha, Sikkim, Tamil Nadu, Tripura (Present report), Uttar Pradesh, West Bengal); Tropical and subtropical regions (5, 13).

Note: This species can be differentiated from others by its long, tough, shiny, silvery-white awns.

3. Perotis indica (L.) Kuntze, Revis. Gen. Pl. 2: 787. 1891; Bor, Fl. Assam 5: 161. 1940 \& Grass. Burma, Ceylon, India, Pakistan: 611. 1960; Patunkar, Grass. Marathwada: 267. 1980; Karthik. et al., Fl. Ind. Enumerat. Monocot.: 216. 1989; Sreek. \& V.J. Nair, Fl. Kerala Grass.: 434. 1991; Kabeer \& V. J. Nair, Fl. Tamil Nadu Grass.: 205. 2009. Anthoxanthum indicum L., Sp. Pl.: 28. 1753. Perotis latifolia Aiton, Hort. Kew. ed. 1: 85. 1789; Hook. f., Fl. Brit. India 7: 98. 1896. Fig. 3, Image 3.

Description: Annual, tufted. Culms geniculate, 25-60 $\mathrm{cm}$ high, glabrous. Leaf-blades lanceolate to ovate, $1.5-4 \times$ ca. $1 \mathrm{~cm}$, amplexicaul at base, tapering into an acute tip, glabrous; ligule very minute, membranous; leaf sheaths loose, glabrous. Inflorescence spike-like terminal raceme, up to $20 \mathrm{~cm}$ long; raceme homogamous. Spikelets linear-lanceolate, $0.8-1.2 \mathrm{~cm}$ long, numerous, solitary, 2-awned, shortly pedicelled, falling entire. Glumes more or less similar, almost as long as the length of spikelet. Lower glume ca. $2 \times 0.5$ $\mathrm{mm}$, mid nerve prominent, chartaceous, awn 6-10 $\mathrm{mm}$, starting from the tip of glume, barbellate. Upper glume 1.8-2 × 0.3-0.5 mm, chartaceous; awned. Lemma lanceolate, $0.8-1 \mathrm{~mm}$ long, acute at apex, membranous, hyaline. Palea similar to lemma. Lodicules 2, hyaline. Stamens 3, ca. $0.5 \mathrm{~mm}$ long. Pistil 0.7-1 mm; styles 2; stigma plumose.
Habitat: Along roadside on sandy soil, near forest floor.

\section{Flowering \& Fruiting: June-November.}

Specimens examined: India, Tripura, Sepahijala district, way to Agartala-Kamalasagar Road, near Nimbutali, $23^{0} 719989$ N, $091^{0} 220685$ E, 10.07.2017, S. Ghosh, 12837, fl.; South district, Rajnagar, near Butterfly park, $23^{0} 268706$ N, $091^{0} 397846$ E, 02.11.2017, S. Ghosh, 12924, fl.

Distribution: India (Andhra Pradesh, Assam, Bihar, Goa, Karnataka, Kerala, Madhya Pradesh, Maharashtra, Odisha, Tamil Nadu, Tripura (Present report), Uttar Pradesh, West Bengal); South-east Asia, Nepal, Sri Lanka (13).

4. Phalaris minor Retz., Observ. Bot. 3: 8. 1783; Hook. f., Fl. Brit. India 7: 221. 1896; Bor. Grass. Burma, Ceylon, India, Pakistan: 616. 1960; Karthik. et al., Fl. Ind. Enumerat. Monocot.: 247. 1989; Shukla, Grass. N.E. India: 368. 1996; Kabeer \& V. J. Nair, Fl. Tamil Nadu Grass.: 84. 2009. Fig. 4, Image 4.

Description: Annuals, tufted, terrestrial to partly marshy. Culms erect, 21-45 cm high. Leaf blades linear-lanceolate, $4-11 \times 0.3-0.8 \mathrm{~cm}$, acuminate at apex; ligules 2-5 mm long, membranous, white; leaf sheath more or less ribbed. Inflorescence contracted panicle, cylindrical or ovoid, $1.5-4 \mathrm{~cm}$, greenish white. Spikelets ovate-elliptic, 4-5.5 × ca. $3 \mathrm{~mm}$, laterally compressed, greenish white; pedicels 1-2 mm long. Glumes similar, well-developed, broadly winged, as long as spikelets, exceeding florets, elliptic-lanceolate, 4-5 mm long, acute at apex, chartaceous, hyaline, 3nerved, midrib keeled; keel broadly winged, finely serrulate, persistent. Florets 2; lower floret reduced to minute lemma, upper floret well developed, bisexual. Lower lemma linear, ca. $1.5 \times 0.3 \mathrm{~mm}$, scale-like, sterile, surface ciliate. Lower palea absent. Upper lemma broadly ovate, ca. $3 \times 2 \mathrm{~mm}$, chartaceous to subcoriaceous, acute at apex, midnerve prominent, keeled towards middle, shiny, pubescent, ciliate at margin. Upper palea ovate-lanceolate, ca. $2.5 \times 0.7$ $\mathrm{mm}$, acute at apex, chartaceous, keeled towards middle, ciliate on keels and mid-portion, greenish. Stamens 3; anthers 1-2 mm long. Pistil ca. $2.5 \mathrm{~mm}$ long; style hyaline; stigma plumose.

Habitat: Along roadside waste land, usually prefers swampy localities.

\section{Flowering \& Fruiting: February-May.}

Specimen examined: India, Tripura, West district, Way to ILS Hospital road, $23^{\circ} 866080 \mathrm{~N}, 091^{\circ} 285860 \mathrm{E}$, 02.03.2019, S. Ghosh, 13036, fl.

Distribution: India (Andhra Pradesh, Arunachal Pradesh, Assam, Bihar, Himachal Pradesh, Kerala, Maharashtra, Meghalaya, Odisha, Sikkim, Tamil Nadu, Tripura (Present report), Uttar Pradesh, West Bengal); Mediterranean regions, Baluchistan, Northwestern Himalayas $(5,13)$.

Note: This species is very rare in the habitat and found in very few numbers only.

5. Pseudoraphis brunoniana (Griff.) Pilg. in Notizbl. Bot. Gart. Berlin-Dahlem 10: 210. 1928 ; Bor, Fl. Assam 5: 292. 1940 \& Grass. Burma, Ceylon, India, Pakistan: 


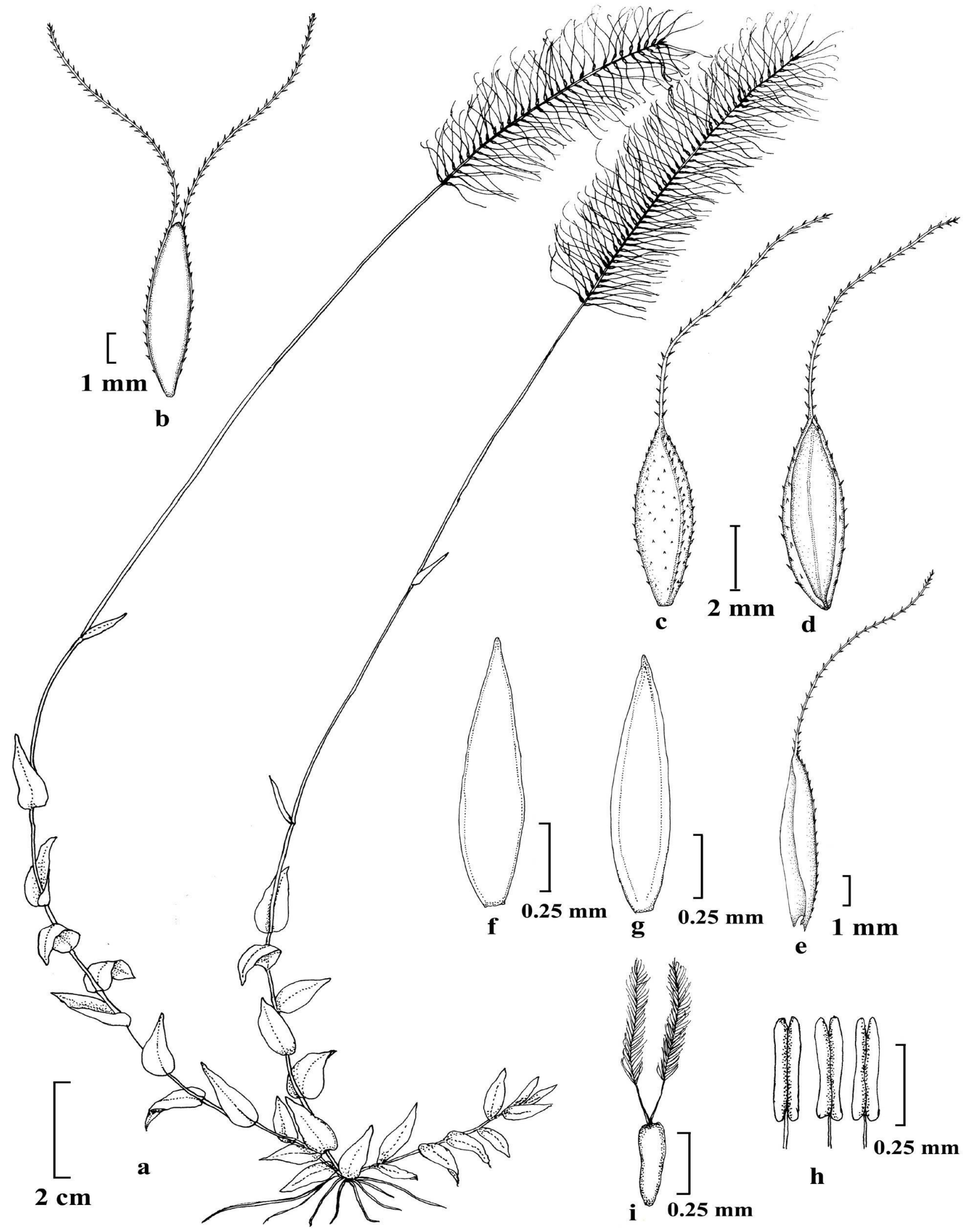

Fig. 3. Perotis indica (L.) Kuntze

a. Habit; b. Single spikelet; c. Lower glume (abaxial view); d. Lower glume (adaxial view); e. Upper glume (side view); f. Lemma (abaxial view); g. Palea (abaxial view); h. Stamens; i. Pistil.

353. 1960; Karthik. et al., Fl. Ind. Enumerat. Monocot.: 253. 1989. Panicum brunonianum Griff. in J. Asiat. Soc. Bengal 5: 547. 1836. Chamaeraphis spinescens var. brunoniana (Griff.) Hook. f., Fl. Brit. India 7: 62. 1896. Fig. 5, Image 5.
Description: Annuals, stoloniferous, mat-forming, aquatic to semi-aquatic plant. Culms ca. $45 \mathrm{~cm}$ high; rooting from lower nodes. Leaf blades linearlanceolate, ca. $5.5 \times 0.5 \mathrm{~cm}$, tapering towards the tip; ligule membranous, ciliolate. Inflorescence panicle, 


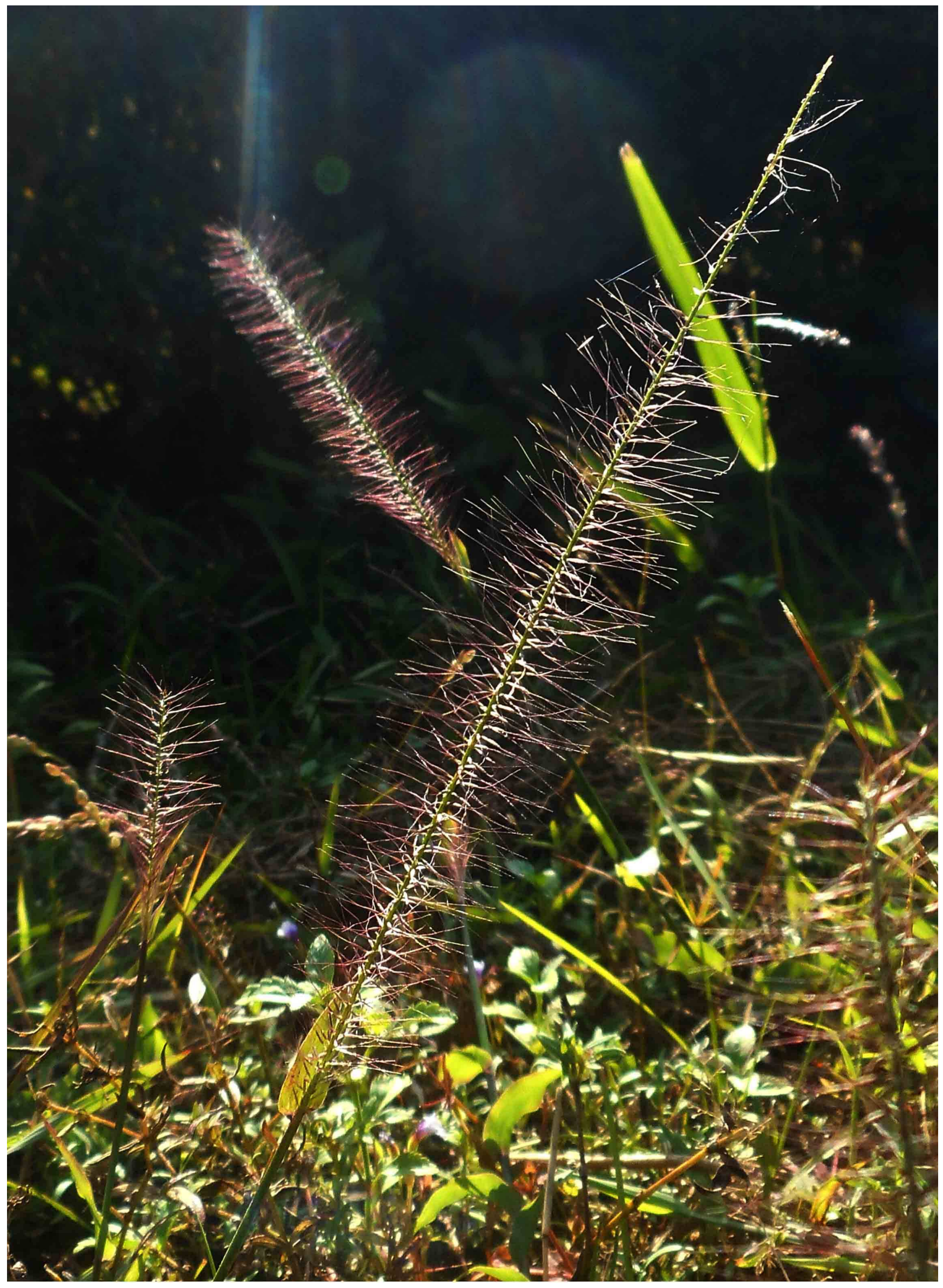

Image 3. Perotis indica (L.) Kuntze 


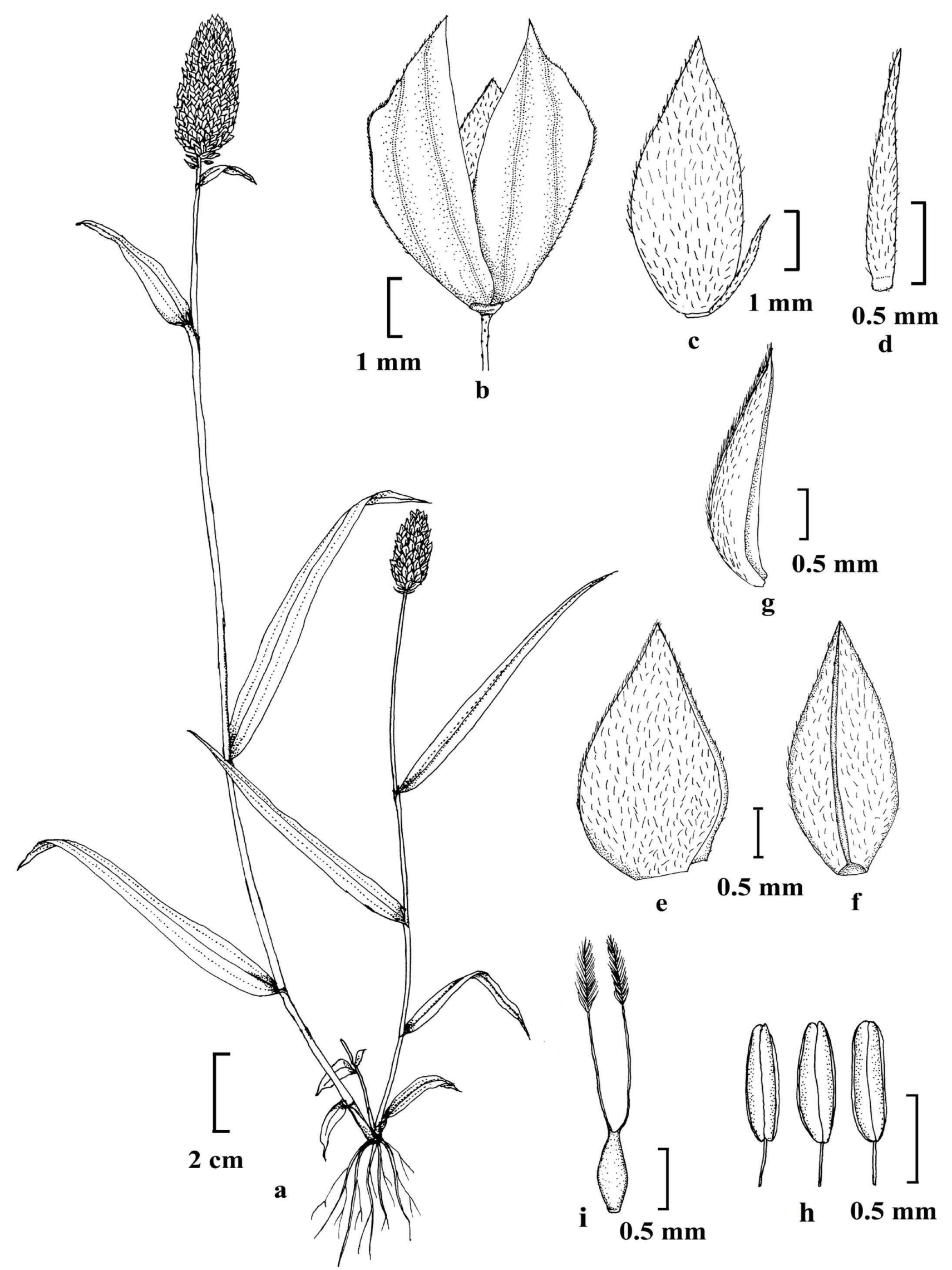

Fig. 4. Phalaris minor Retz.

a. Habit; b. Single spikelet showing broadly winged glumes; c. Upper floret with reduced lower lemma; d. Reduced lower lemma; e. Upper lemma (side view); f. Upper lemma (adaxial view); g. Upper palea (side view); h. Stamens; i. Pistil.

6-10 cm long, rachis scabrous. Spikelets linear- formed by prolongation of the branches, 1-2 lanceolate, $7.5-9 \times$ ca. $1.2 \mathrm{~mm}$, subtended by a bristle, (sometimes 3), greenish white, falling entire; pedicel 


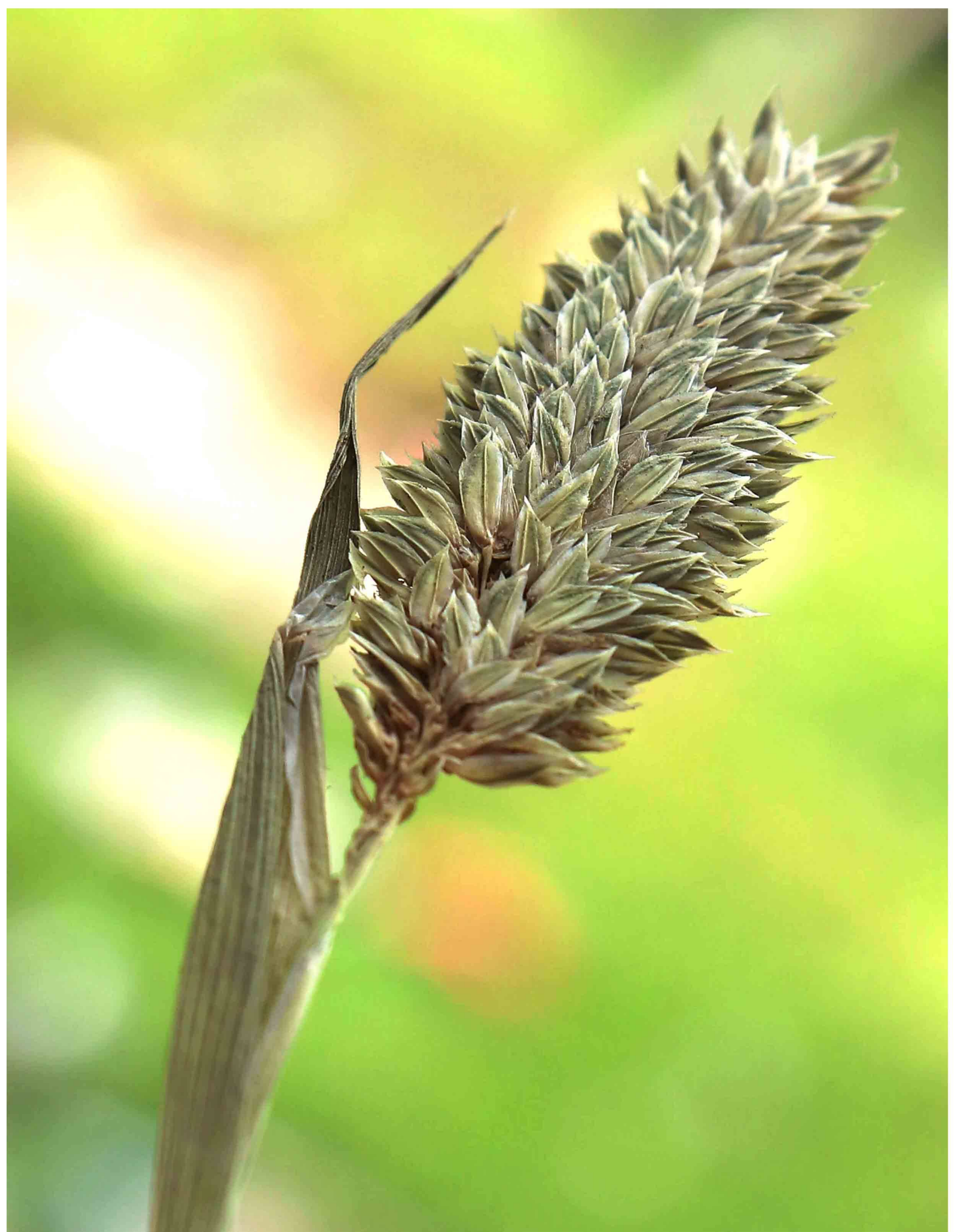

Image 4. Phalaris minor Retz.

short, serrulate. Lower glume orbicular, $0.8-1 \mathrm{~mm}$, faintly 3-nerved, whitish. Upper glume lanceolate, 7.5-9 × 1-1.2 mm, 7-nerved, as long as spikelet, margins infolded, spinulose on nerves and margins.
Florets 2; lower empty, upper bisexual. Lower lemma lanceolate, 6-7.5 × ca. $1 \mathrm{~mm}, 7-$ nerved, sparsely spinulose on apex and margins. Lower palea oblonglanceolate, 4-5 × ca. $1 \mathrm{~mm}$, faintly 2-nerved. Upper 


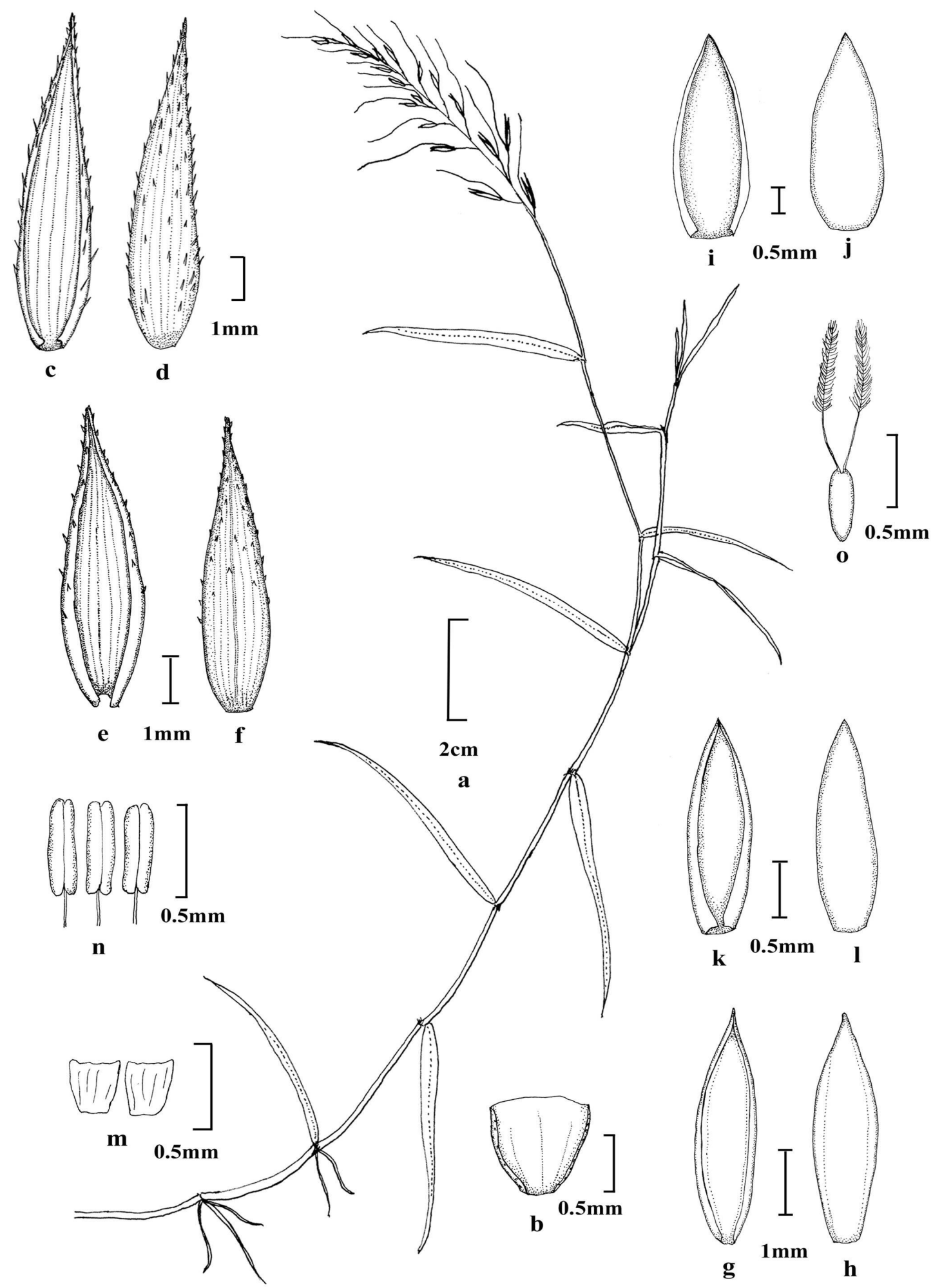

Fig. 5. Pseudoraphis brunoniana (Griff.) Pilg.

a. Habit; b. Lower glume; c. Upper glume (adaxial view); d. Upper glume (abaxial view); e. Lower lemma (adaxial view); f. Lower lemma (abaxial view); g. Lower palea (adaxial view); h. Lower palea (abaxial view); i. Upper lemma (adaxial view); j. Upper lemma (abaxial view); k. Upper palea (adaxial view); l. Upper palea (abaxial view); m. Lodicules; n. Stamens; o. Pistil.

lemma linear-oblong, $2-3 \times$ ca. $0.5 \mathrm{~mm}$, margin infolded, smooth. Upper palea oblong, ca. $2 \times 0.5 \mathrm{~mm}$, subcoriaceous. Lodicules 2, $0.5-0.7 \mathrm{~mm}$, hyaline.
Stamens 3; ca. 2 mm long. Pistil 1.5-2 mm long; styles 2, filiform; stigma plumose.

Habitat: Marshy or semi-aquatic fields. 


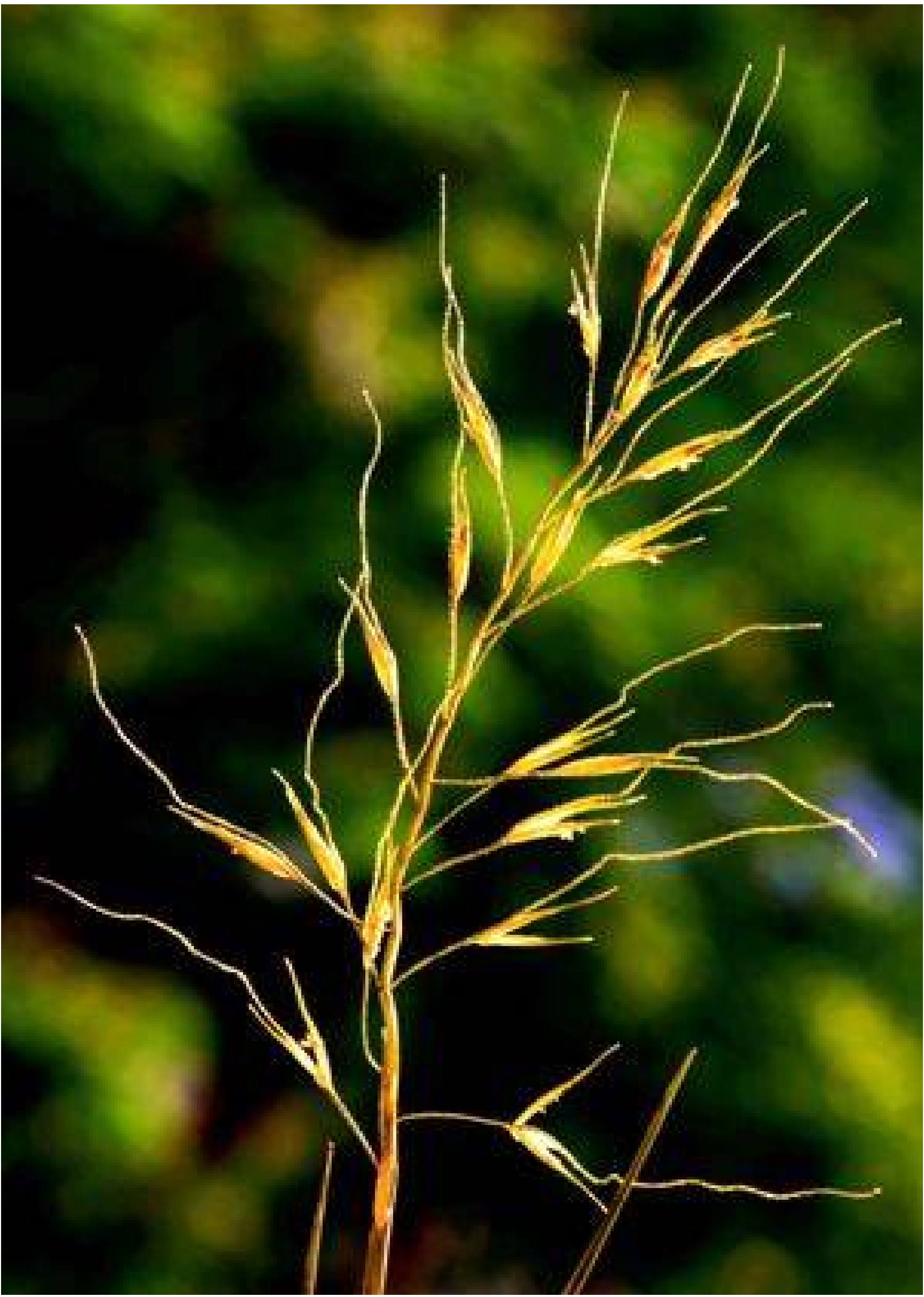


Flowering \& Fruiting: April-July.

Specimen examined: India, Tripura, West district, West Pratapgarh, near hanging bridge, $23^{\circ} 819480 \mathrm{~N}$, 0910282696 E, 07.07.2017, S. Ghosh, 12827, fl..

Note: Lower part of this plant remains submerged in shallow water. The species was found growing along with Panicum repens and Hymenachne sp.

Distribution: India (Assam, Bihar, Madhya Pradesh, Manipur, Nagaland, Odisha, Tripura (Present report), West Bengal); South-east Asia, Sri Lanka, Australia (5, 13).

\section{Conclusion}

Of the five genera recorded here, three genera, namely Eriochloa Kunth, Heteropogon Pers. and Pseudoraphis Griff. belong to the subfamily Panicoideae and other two genera Perotis Aiton and Phalaris L. are members of the subfamily Chloridoideae and Pooideae respectively.

Representative species of the genera Heteropogon, Perotis and Phalaris were found to grow in terrestrial habitats in the study area whereas, that of Eriochloa and Pseudoraphis were found in semi-aquatic and aquatic habitats. Field studies showed that out of the five species, only Perotis indica was found common in the state. Other four species were found to be confined to some particular localities and were not common in the study area.

\section{Authors' contributions}

SG carried out field visits, collection, analysis and identification, illustration preparation and initial manuscript writing. DB supervised the whole work, incorporated his suggestions, corrected the manuscript and finally submitted the manuscript for publication.

\section{Acknowledgements}

The authors are thankful to the Head, Department of Life Science and Bioinformatics, Assam University, Silchar for providing facilities. They are also thankful to the Head-in-Charge of ASSAM and CAL, Botanical Survey of India for giving permission to consult the herbaria and library. They also extend their sincere thanks to the Forest Department of Tripura for giving permission to conduct field surveys in the state. The first author sincerely acknowledges the financial support provided by the UGC, New Delhi under National Fellowship for Other Backward Classes (NFOBC) Scheme.

\section{Competing interests}

The authors declare no competing interests.

\section{References}

1. Yadav SR. Know your grass genera through hand lens. Shivaji University, Kolhapur; 2010

2. Mabbarley DJ. Mabberley’s Plant-Book: A portable dictionary of plants, their classification and uses. Fourth Edition. Cambridge University Press, London. 2017

3. Karthikeyan S, Jain SK, Nayar MP, Sanjappa M. Florae Indicae Enumeratio: Monocotyledonae. Botanical Survey of India, Flora of India; 1989. Series 4

4. Arisdason W, Lakshminarasimhan P. Status of Plant Diversity in India: an overview. ENVIS Centre on Floral Diversity [Internet]. Botanical Survey of India, Kolkata, India [cited 2020 Apr 8]. Available from: http://www.bsienvis.nic.in/Database/Status_of_Plant_Diversity_ in_India_17566.aspx

5. Shukla U. Grasses of North-Eastern India. Scientific Publishers Jodhpur; 1996

6. Deb DB. The Flora of Tripura State. vol. 2. Today and Tomorrow's Printers and Publishers, New Delhi. 1983;486-547

7. Datta BK, Saha R, Roy M, Majumder K. Grasses of West Tripura District, Tripura, India. Pleione. 2008;2(1):98-105

8. Bhowmik S, Datta BK. The first report on the occurrence of pseudo-vivipary from Pennisetum polystachyon (L.) Schult. (Poaceae) from India. J New Biol Rep. 2014;3(2):137-40

9. Ghosh S, Bhattacharyya D. Five new additions to the grass flora of Tripura State, India. J Threat Taxa. 2018;10(3):11484 92._http:// doi.org/10.11609/jott.3461.10.3.11484-11492

10. Bor NL. Gramineae. In: Kanjilal et al., Flora of Assam, Vol. 5 , Government Press, Shillong; 1940

11. Bor NL. The Grasses of Burma, Ceylon, India and Pakistan (Excluding Bambuseae). Pergamon Press, London; 1960

12. Clayton WD, Renvoize SA. Genera Graminum. Royal Botanic Gardens, Kew, London; 1986

13. Kabeer KAA, Nair VJ. Flora of Tamil Nadu - Grasses. BSI, Calcutta; 2009

14. IPNI. International Plant Names Index [Internet]. The Royal Botanic Gardens, Kew, Harvard University Herbaria \& Libraries and Australian National Botanic Gardens [cited 2020 Apr 8]. Available from: http://www.ipni.org

15. IUCN. The IUCN Red List of Threatened Species, 2020. Version 2020-1[Internet]. [cited 2020 Apr 8]. Available from: http://www.iucnredlist.org 